\title{
RED FLAGS IN A PROTOTYPICAL CASE OF VZV-VASCULOPATHY.
}

Silvio Piffer ${ }^{1}$, Monica Margoni1, Valentina Poretto', Sabrina Marangoni1, Maria Pellegrini1, Umberto Rozzanigo², Bruno Giometto1.

1) Department of Neurological Disorders, Neurology, Santa Chiara Hospital, Trento, Italy. 2) Department of Radiology, Neuroradiology, Santa Chiara Hospital, Trento, Italy. Correspondence to silviopiffer@yahoo.it.

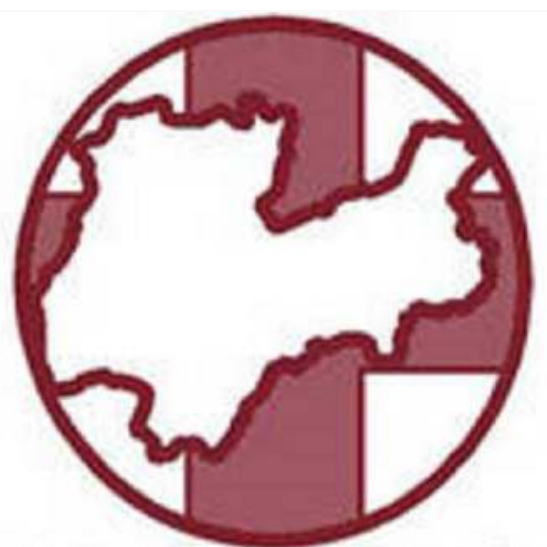

Azienda Provinciale per i Servizi Sanitari Provincia Autonoma di Trento

\section{Introduction.}

Zoster is a well-known risk factor for stroke. Varicella zoster virus (VZV) can infect cerebral arteries after re-activation. VZV-vasculopathy is challenging because of heterogeneous and evolving phenotype and possible unfavourable course without prompt treatment. We report a prototypical case.

\section{Case Report.}

History.

A 84-year-old healthy and immunocompetent woman referred to emergency department because of confusional state during fever. In the emergency room she presented a tonic-clonic seizure of left limbs followed by persistent vigilance impairment, left side hemiparesis with anesthesia and right-forced gaze version.

During previous month she suffered right ear pain and deafness without local rash and progressive cognitive impairment.

\section{Investigations.}

- Head CT scan: right medial frontal hypo-density.

- EEG: background slow activity with frontal sharp waves. (A)

- Otoscopy: a recent right middle ear acute otitis.

- Carotid and heart ultrasound, ECG: unremarkable.

- Blood exams: unremarkable except for mild elevation of C-reactive protein and leucocytes.

- Cerebrospinal fluid (CSF) standard exam: 3 lymphocytes and normal protein and glucose concentrations

- Polymerase chain reaction (PCR) for neurotropic viruses positive for VZV-DNA.

- Brain MRI: hyper intensity in the territory of right anterior cerebral artery (ACA) on DWI and FLAIR sequences (B-C') suggestive for acute ischemic stroke associated with intralesional focal hypo intensity on GRE sequence ( $\left.D, D^{\prime}\right)$ suggestive for small intralesional hemorrhagic foci. After gadolinium injection vessel irregularities of the right ACA on angio $M R I(F)$ and diffuse post-gadolinium leptomeningeal enhancement on $T 1$ sequences ( $\left.E, E^{\prime}\right)$.

- Intracranial vessel angiography: irregularities of right anterior cerebral artery tract 1 and 2. (G)

Treatment.

Parenteral Acyclovir (3 ws), Methylprednisolone (5 ds) and Levetiracetam 3g/d, associated to long-term Aspirin 0.1g/d.

Clinical course.

At three-months follow-up, patient well oriented with mild gaze impairment and left side hemiparesis.
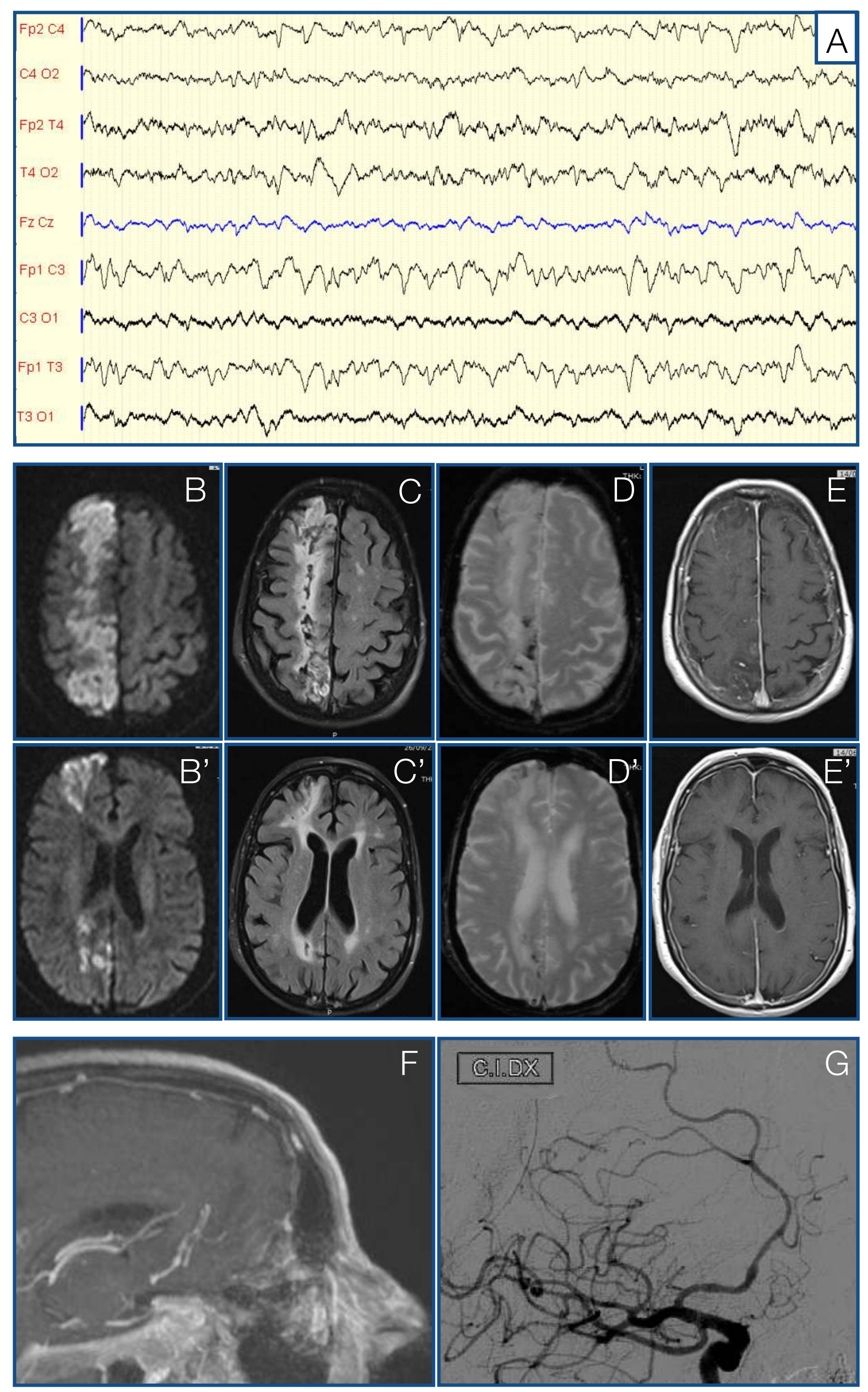

\section{Conclusions.}

We diagnosed acute ischemic stroke due to focal vasculitis of the right ACA (VZV-vasculopathy) in a patient with VZV meningo-encephalitis. VZV-vasculopathy should be suspected not only in stroke patients with history of Zoster but also in atypical stroke presentations associated with a recent evolving cranial neuralgia or encephalopathy. Immunosuppression is not a prerequisite especially in older patients. VZV-DNA search on CSF should be performed. 